\title{
Tratamento odontológico durante a gestação: conhecimentos e percepções de estudantes de Odontologia
}

\author{
Raíssa Costa Ferreira Elias*; Paula Molina Nogueira**; Mara Vasconcelos***; Lívia Guimarães \\ $\operatorname{Zina}^{* * * * *}$ \\ * Estudante, Faculdade de Odontologia, Universidade Federal \\ de Minas Gerais \\ ** Cirurgiã-dentista, estudante do Mestrado Profissional em \\ Odontologia em Saúde Pública, Faculdade de Odontologia, \\ Universidade Federal de Minas Gerais \\ *** Doutora em Odontologia Social, Professora Associada, \\ Departamento de Odontologia Social e Preventiva, Faculdade \\ de Odontologia, Universidade Federal de Minas Gerais \\ **** Doutora em Odontologia Social e Preventiva, Professora \\ Adjunto, Departamento de Odontologia Social e Preventiva, \\ Faculdade de Odontologia, Universidade Federal de Minas \\ Gerais
}

Recebido em 06/11/2017. Aprovado em 28/06/2018.

\begin{abstract}
RESUMO
O tratamento odontológico durante a gestação envolve mitos e incertezas, perpetuados pelo medo dos profissionais em atender gestantes. Poucos cursos de odontologia no Brasil possuem algum tipo de disciplina, projeto, programa ou ação que proporcione a capacitação do graduando para o cuidado dessa paciente. O objetivo deste trabalho foi avaliar o conhecimento dos acadêmicos de um curso de graduação em Odontologia sobre o atendimento odontológico à gestante e conhecer as necessidades desse grupo quanto ao aprendizado desse tema. Foi aplicado um questionário semiestruturado a todos os alunos matriculados no segundo semestre de 2016 na Faculdade de Odontologia da Universidade Federal de Minas Gerais. O questionário abordou questões como conhecimentos e expectativas sobre o atendimento odontológico à gestante. Os resultados foram analisados pela técnica de Análise de Conteúdo, e calculadas estimativas de prevalência. Dentre os 303 alunos que responderam ao questionário, apenas $14 \%$ atenderam gestantes durante a graduação. Somente $43 \%$ dos estudantes consideraram-se preparados para atender gestante, e $62 \%$ relataram ter recebido alguma orientação sobre o pré-natal odontológico durante a graduação, não existindo um conteúdo formal associado a alguma disciplina. Quanto à técnica, os principais problemas encontrados referiram-se ao desconhecimento sobre o uso de medicamentos, anestésicos e procedimentos permitidos. Em conclusão, os estudantes apresentaram deficiências no conhecimento sobre o atendimento odontológico à gestante, e demonstraram interesse em melhorar seu aprendizado nessa área. $\mathrm{O}$ ensino da graduação não está preparando adequadamente os alunos para o pré-natal odontológico, reforçando o ciclo de medos e adágios que envolvem esse cuidado.
\end{abstract}

Descritores: Gestante. Assistência Odontológica. Conhecimento. Estudante. Educação em Odontologia. 


\section{INTRODUÇÃO}

A gestação é um período muito delicado e que inspira vários cuidados com a alimentação, ganho de peso, a manutenção da saúde geral e bucal, ingestão de medicamentos, exposição a fatores de risco ambientais, alterações emocionais, dentre outros ${ }^{1}$. Nessa fase, inúmeras alterações ocorrem no corpo da mulher, tanto fisiológicas quanto psicológicas, sendo que algumas dessas mudanças vão influenciar diretamente na sua condição de saúde bucal ${ }^{1-4}$.

As alterações observadas nessa fase representam processos fisiológicos normais decorrentes da preparação do organismo feminino para a geração do bebê. Observam-se, com frequência, mudanças no aspecto gengival das pacientes, com uma tendência ao agravamento da gengivite, tornando-se mais perceptível frente à presença de irritantes locais ${ }^{2,3}$. A possibilidade de maior proliferação de micro-organismos na cavidade oral durante a gestação facilita a colonização pelos periodontopatógenos ${ }^{4}$.

A prevalência da doença periodontal, baseada em observações clínicas, tem variado entre os trimestres gestacionais, atingindo entre 30 a $50 \%$ das mulheres grávidas ${ }^{5-8}$. Há evidências científicas de que a doença periodontal na gestação atua como um dos fatores predisponentes para o parto prematuro, apesar de restrito a determinados grupos populacionais ${ }^{8}$. Da mesma forma, a dor de dente é bastante recorrente durante a gestação, e tem a doença cárie como seu principal fator determinante ${ }^{9}$.

Toda gestante deveria fazer o pré-natal odontológico, em virtude de tais alterações fisiológicas e riscos temporários a problemas bucais. Todavia, um grande dilema é enfrentado nesse contexto: o medo odontológico. Estudos têm demonstrado que tanto as gestantes justificam ter medo do tratamento odontológico, quanto os próprios cirurgiões-dentistas recusamse a atender pacientes gestantes ${ }^{10-12}$.

Apesar do atendimento odontológico à paciente gestante fazer parte do protocolo de cuidados de pré-natal no Sistema Único de Saúde ${ }^{13}$, e das inúmeras evidências acerca da segurança dos procedimentos odontológicos na gravidez ${ }^{14}$, muitos profissionais da saúde bucal ainda têm receio de atender esse grupo ${ }^{11}$. O desconhecimento e despreparo são as principais causas da recusa por parte dos cirurgiõesdentistas ${ }^{10-12}$. Em contrapartida, na literatura, há escassos relatos de instituições de ensino que ofertam, durante os cursos de graduação em Odontologia, conteúdos voltados para o atendimento odontológico da gestante, assim como a oportunidade de realizar procedimentos clínicos nessas pacientes ${ }^{15}$. Diante disso, compreende-se que uma das principais formas de enfrentar o problema é investir na formação profissional ainda na graduação.

Diversos estudos avaliaram o conhecimento e atitudes de cirurgiões-dentistas e pessoal auxiliar frente ao cuidado odontológico da gestante, porém pouco se conhece sobre a percepção do estudante da área ${ }^{16}$. Essa avaliação é importante para fomentar a discussão sobre a formação profissional e subsidiar estratégias de capacitação de alunos nos cursos de graduação, preparando-os para o atendimento integral e interdisciplinar da mulher no período reprodutivo. Assim, este estudo objetivou avaliar o conhecimento dos acadêmicos de um curso de graduação em odontologia sobre o atendimento odontológico à gestante e conhecer as necessidades desse grupo quanto ao aprendizado desse tema.

\section{METODOLOGIA}

Este é um estudo transversal, de caráter retrospectivo, e abordagem quali-quantitativa. Foi conduzido com os alunos do curso de 
graduação em Odontologia da Universidade Federal de Minas Gerais (UFMG). O curso contempla 10 períodos/semestres formativos. Foram elegíveis para este estudo todos os 480 alunos que estavam cursando entre o quarto e o décimo período da graduação. Esses períodos foram selecionados porque envolvem a iniciação às atividades clínicas, cujos pacientes são adolescentes e adultos (e, portanto, inclui a mulher no período reprodutivo). Os participantes deveriam entregar o Termo de Consentimento Livre e Esclarecido devidamente assinado. Os dados foram coletados no segundo semestre de 2016.

Para a coleta de dados, foi elaborado um questionário semiestruturado autoaplicável (com perguntas abertas e fechadas), envolvendo questões como caracterização dos sujeitos de pesquisa e conhecimentos sobre o pré-natal odontológico. As questões abertas foram utilizadas para identificar como se deu a experiência dos alunos no atendimento de gestantes, e as facilidades e dificuldades encontradas por eles nesse atendimento. Optouse por não utilizar respostas pré-categorizadas para essas questões, pois não se sabia ao certo quais seriam as possibilidades de resposta, visto não haver até o momento de elaboração do instrumento desta pesquisa estudos publicados com essa temática ou instrumentos já validados. Dessa forma, as questões abertas foram utilizadas com o intuito de identificar quais seriam as possíveis respostas dos alunos. Para um estudo futuro, será possível categorizar as alternativas de respostas para essas questões, com base nos resultados obtidos no presente estudo.

$\mathrm{O}$ instrumento foi testado em um estudo piloto com alunos de um projeto de extensão da mesma faculdade, o qual contempla estudantes de diferentes períodos da graduação. Para isso, foram utilizadas questões cognitivas para avaliar a capacidade do instrumento de ser compreensível para seus respondentes, através do referencial teórico da metodologia de survey ${ }^{17}$. Os respondentes do estudo piloto foram excluídos da amostra final do estudo. Uma pesquisadora (RCE) foi treinada no estudo piloto, e o questionário reelaborado após avaliação da confiabilidade das respostas. Por fim, o questionário foi entregue pessoalmente aos estudantes em sala de aula, ou antes do atendimento clínico, após autorização do professor presente na aula. Os estudantes levaram, em média, 10 minutos para responder ao instrumento de pesquisa.

Foi elaborada uma máscara específica para digitação dos dados no software Epi Info ${ }^{\text {TM }} 7$ (versão 7.2.1.0, CDC, Atlanta, GA, USA). Os dados coletados foram codificados, conferidos, digitados e processados neste programa.

A análise qualitativa foi utilizada nas questões que abordaram a experiência, dificuldades e facilidades no atendimento de gestantes e, portanto, só foram respondidas pelos alunos que atenderam gestantes durante o curso de graduação. Apesar do número elevado de respondentes, essa análise qualitativa foi útil para a identificação de categorias de respostas para o assunto estudado. As respostas das questões abertas do questionário foram transcritas $\mathrm{e}$ analisados pelo método de Análise de Conteúdo ${ }^{18}$. Segundo Bardin ${ }^{18}$, esta técnica pode ser conceituada como um conjunto de técnicas de análise das comunicações, visando, por procedimentos sistemáticos e objetivos de descrição do conteúdo das mensagens, obter indicadores quantitativos ou não, que permitam a inferência de conhecimentos relativos às condições de produção/recepção (variáveis inferidas) das mensagens.

Dentre as técnicas de Análise de Conteúdo, foi realizada, neste estudo, a análise categorial, funcionando através de operações de desmembramento do texto em unidades e em 
categorias segundo reagrupamentos analógicos. Os resultados foram então descritos conforme a distribuição nas categorias e apresentadas as falas dos sujeitos.

Para a análise quantitativa das questões fechadas do questionário, foram calculadas as estimativas de prevalência, e realizadas análises estatísticas bivariadas, ao nível de significância de 5\%. Como variável dependente, foi criada a variável "conhecimento", com a classificação dos estudantes em "com conhecimento adequado" e "sem conhecimento adequado", a partir das respostas obtidas com as questões sobre o que é permitido no tratamento odontológico da gestante. Foi analisada a associação dessa variável com o período do curso em que o estudante estava matriculado, os procedimentos realizados em gestantes, a dificuldade no atendimento, e a orientação recebida durante o curso de graduação. Foram utilizados para isso os softwares Epi Info $^{\mathrm{TM}}$ versão 7.2.1.0 e SPSS ${ }^{\mathrm{TM}}$ versão 22.0.

Este estudo se enquadra na modalidade de pesquisa de risco mínimo, de acordo com a resolução 466/2012 do Conselho Nacional de Saúde, relativa à pesquisa em seres humanos. A pesquisa obteve aprovação do COEP (CAAE 57662216.6.0000.5149 - parecer número 1.717.344), e todos os alunos que aceitaram participar do estudo assinaram o Termo de Consentimento Livre e Esclarecido.

\section{RESULTADOS}

Trezentos e três alunos responderam aos questionários, correspondendo a $63,1 \%$ do universo estudado. Dentre os motivos para a perda amostral destacam-se os estudantes que não estavam presentes no momento da entrega do questionário e com os quais não foi obtido contato (foram feitas três tentativas, incluindo busca ativa) e aqueles que se recusaram a participar do estudo.
A distribuição dos estudantes participantes entre os períodos avaliados foi equitativa, com exceção do nono período, cuja perda amostral foi maior. Participaram do estudo $23 \%$ dos estudantes do décimo período, $15 \%$ do período, $14 \%$ do quarto, $14 \%$ do quinto, $12 \%$ do sexto, $14 \%$ do oitavo e $8 \%$ do nono período.

A maioria dos estudantes $(84,0 \%)$ não atendeu gestante durante o curso de graduação, e uma pequena parcela $(2,0 \%)$ não soube informar se já havia atendido ou não (pela dúvida da paciente estar grávida ou por não saberem). $\mathrm{O}$ atendimento, quando aconteceu, se deu, majoritariamente, nos últimos períodos, apesar da sua distribuição ter sido proporcional ao número de alunos respondentes em cada período.

A figura 1 apresenta os procedimentos que foram realizados nas gestantes atendidas nas clínicas do curso de graduação, destacando-se as restaurações dentárias (37\%) e as orientações de higiene e cuidados com a saúde bucal (30\%).

$\mathrm{Na}$ análise das questões abertas que abordaram a experiência do atendimento clínico da gestante (14\% dos respondentes), emergiram três categorias: experiências positivas, negativas ou indiferença. As respostas dos participantes são apresentadas no quadro 1. As falas referentes às dificuldades encontradas nesse atendimento relatadas por $28 \%$ dos alunos que atenderam gestantes - foram agrupadas em três categorias, que envolveram os procedimentos odontológicos em geral, o posicionamento na cadeira odontológica e a insegurança no exercício da prática clínica (quadro 2 ).

Quando indagados sobre a formação para o tratamento odontológico da gestante, mais da metade $(57 \%)$ dos estudantes afirmaram não se sentirem preparados para realizar esse tipo de atendimento. Do total, $62 \%$ relataram ter recebido orientação sobre o pré-natal odontológico no curso de graduação, majoritariamente em disciplinas de cirurgia e 
clínicas. Quando questionados sobre o interesse em participar de atividades de ensino que envolvessem o pré-natal odontológico, como em um projeto de extensão, $83 \%$ responderam que gostariam de participar, representando assim uma demanda reprimida.

A tabela 1 apresenta a conduta terapêutica recomendada para o tratamento odontológico da gestante, de acordo com o conhecimento dos alunos. Uma grande parcela deles não soube responder a uma ou mais perguntas. Para aqueles que o fizeram, de forma geral, o tratamento da gestante pode envolver, de preferência no segundo trimestre de gestação, a realização de restaurações, raspagens e polimentos coronários, a prescrição de amoxicilina e paracetamol (quando necessários), utilizando-se o anestésico lidocaína, e podendo fazer uso de radiografia odontológica.

Não foi encontrada associação estatisticamente significativa entre o conhecimento dos alunos sobre o tratamento odontológico e o período do curso em que o estudante estava matriculado, os procedimentos realizados em gestantes, a dificuldade no atendimento, e a orientação recebida durante o curso de graduação (Teste qui-quadrado, $\mathrm{p}>0,05$ ).

\section{DISCUSSÃO}

Este estudo avaliou o conhecimento dos alunos de Odontologia da UFMG sobre o cuidado odontológico à gestante. A formação profissional do estudante está aquém do necessário para que ele atue de forma segura no pré-natal odontológico.

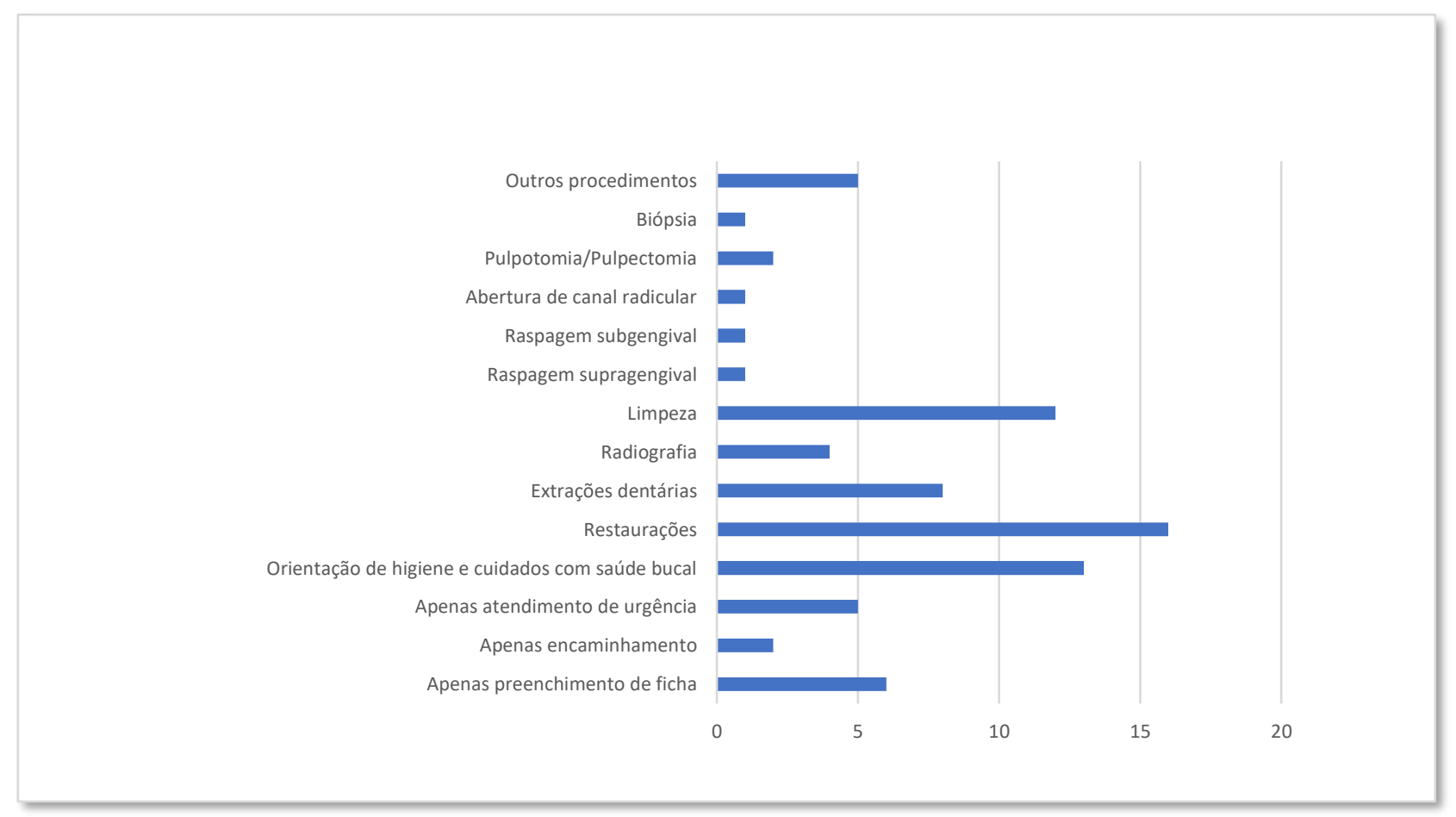

Figura 1. Principais procedimentos realizados pelos estudantes em gestantes durante atendimento clínico na UFMG 
Quadro 1. Percepção dos estudantes sobre a experiência de atender gestantes nas clínicas da Faculdade de Odontologia da UFMG

\begin{tabular}{|cl|}
\hline $\begin{array}{c}\text { No. de } \\
\text { respostas }\end{array}$ & Experiência (categoria temática) \\
25 & 1. Positiva \\
& "Experiência boa, ganhei mais segurança para indicar e realizar tratamento em gestante". \\
& "Pra mim foi bastante proveitosa devido a pouca experiência com gestante. Hoje tenho \\
& uma visão mais ampla de quando pode e não pode intervir". "A experiência é boa porque \\
& aprendemos a lidar com alguns imprevistos e cuidados que temos que ter". \\
& 2. Negativa \\
& "Ruim, pois não tinha conhecimento para atender gestante". "Complexo. Receio de tirar \\
& Radiografia, receio da paciente passar mal, o professor que conduziu o procedimento". \\
& "Senti despreparada, não senti confiança nos professores. Poderia ter sido melhor". \\
& 3. Indiferente \\
& "Normal, atentando para dar algumas informações relevantes para a gestante". "Não vi \\
& diferença". "A experiência foi a mesma de uma não gestante".
\end{tabular}

Quadro 2. Dificuldades relatadas pelos estudantes no atendimento odontológico de gestantes nas clínicas da Faculdade de Odontologia da UFMG

\begin{tabular}{|cl|}
\hline $\begin{array}{c}\text { No. de } \\
\text { respostas }\end{array}$ & Dificuldades (categoria temática) \\
07 & $\begin{array}{l}\text { 1. Procedimentos odontológicos } \\
\text { "Quais procedimentos poderia realizar". "Não sabia das restrições do atendimento". } \\
\text { "Anestesia..." "... radiografia..." "Uma das grávidas atendidas, a primeira, tinha abcesso } \\
\text { e dor associada. Tive dificuldades para prescrever, mas o dentista do Odilon, Júlio, me } \\
\text { ajudou". }\end{array}$ \\
$\begin{array}{l}\text { 2. Posicionamento na cadeira odontológica } \\
\text { "Insegurança quanto o posicionamento da paciente". }\end{array}$ \\
$\begin{array}{l}\text { 3. Insegurança para o atendimento da gestante } \\
02\end{array}$ \\
\end{tabular}

Menos de um quarto dos estudantes atenderam gestantes durante o curso de graduação. É muito comum a gestante procurar atendimento odontológico somente em situações de urgência, proporcionadas pelo sofrimento da dor dentária. Em um estudo conduzido com 740 gestantes no Canadá, constatou-se que cerca de $50 \%$ dessas mulheres não fizeram um pré-natal odontológico, enquanto $23 \%$ procuraram o atendimento apenas por motivo de urgência ${ }^{19}$. A taxa de procura por atendimento odontológica na gestação tem se mostrado muito baixa, em torno de $15 \%{ }^{2}$. Isso se reflete na quantidade muito pequena de alunos que tiveram a oportunidade de atender esse tipo de paciente. Dentre esses acadêmicos, $27 \%$ estavam no último período do curso de graduação. Isso pode ser justificado pelo fato de que nesse período os alunos atendem pacientes na disciplina de Urgências Odontológicas, desenvolvida dentro da própria instituição de ensino, assim como também têm a oportunidade desde o nono período de participarem do projeto de urgência odontológica em um hospital público municipal. Neste projeto de extensão, chama a atenção a grande quantidade de gestantes com dor que procuram o atendimento. Com a baixa procura de gestantes por atendimento odontológico eletivo há de se 
Tabela 1. Conduta terapêutica no atendimento clínico das gestantes, de acordo com os conhecimentos dos estudantes de graduação $(n=303)$

\begin{tabular}{|c|c|c|}
\hline Conduta terapêutica & $\mathbf{n}$ & $\%$ \\
\hline \multicolumn{3}{|l|}{ Anestésico indicado ${ }^{\S}$} \\
\hline Lidocaína & 104 & 34,3 \\
\hline Qualquer anestésico sem vasoconstritor & 21 & 6,9 \\
\hline Prilocaína & 12 & 4,0 \\
\hline Mepivacaína & 07 & 2,3 \\
\hline Articaína & 06 & 2,0 \\
\hline Bupivacaína & 03 & 1,0 \\
\hline Outros & 01 & 0,3 \\
\hline Não sei/não respondeu & 161 & 53,1 \\
\hline \multicolumn{3}{|c|}{ Melhor período gestacional indicado para tratar a gestante $^{\S \S}$} \\
\hline $1^{\mathrm{o}}$. período & 20 & 6,7 \\
\hline $2^{\circ}$. período & 168 & 56,6 \\
\hline $3^{\circ}$. período & 58 & 19,5 \\
\hline Não sei & 51 & 17,2 \\
\hline \multicolumn{3}{|l|}{ Exposição à radiografia odontológica ${ }^{\S \S \S}$} \\
\hline Pode & 174 & 59,1 \\
\hline Não pode & 76 & 26,0 \\
\hline Não sei & 44 & 14,9 \\
\hline \multicolumn{3}{|l|}{ Medicamentos permitidos ${ }^{\S}$} \\
\hline Paracetamol & 136 & 44,9 \\
\hline Amoxicilina & 119 & 39,3 \\
\hline Diclofenaco de Sódio & 34 & 11,2 \\
\hline Metronidazol & 28 & 9,2 \\
\hline Ácido Acetilsalicílico & 18 & 5,9 \\
\hline Tetraciclina & 02 & 0,7 \\
\hline Não sei & 140 & 46,2 \\
\hline \multicolumn{3}{|l|}{ Procedimentos clínicos $^{\S}$} \\
\hline Polimento dentário & 262 & 86,5 \\
\hline Restauração & 227 & 75,0 \\
\hline Raspagem e alisamento coronorradicular & 206 & 68,0 \\
\hline Aplicação tópica de flúor & 168 & 55,4 \\
\hline Preparo e instalação de prótese & 164 & 54,1 \\
\hline Exodontia & 99 & 32,7 \\
\hline Tratamento de Canal & 99 & 32,7 \\
\hline Nenhum & 03 & 1,0 \\
\hline Não sei & 32 & 10,6 \\
\hline
\end{tabular}

${ }^{\S}$ Número total de respostas superior a 303, já que o participante poderia assinalar mais de uma opção; ${ }^{\S}$ Número total de respondentes: 297; ${ }^{\S}$ Número total de respondentes: 294

estabelecer estratégias de propiciar o contato do aluno com essas pacientes, como parcerias institucionais com serviços públicos onde são realizados atendimentos de pré-natal, ou a criação de serviços especializados nas clínicas das faculdades com ações de busca ativa de gestantes no município. No mínimo, o aluno deve ser instrumentalizado, teoricamente, para o cuidado do pré-natal nas disciplinas correntes do curso. Soma-se a isso o interesse relatado pelos graduandos em participarem de atividades de ensino que envolvessem o pré-natal odontológico, justificando a necessidade de uma ação educacional.

Sobre os procedimentos realizados pelos alunos no atendimento das gestantes, chama a 
atenção o fato de serem estes procedimentos menos complexos, como restaurações, orientações de higiene e cuidados com a saúde bucal e profilaxia. Tais cuidados menos invasivos podem estar relacionados à baixa procura das pacientes ou mesmo à recusa dos profissionais em executar determinados tratamentos. Curioso notar que, apesar da literatura demonstrar que um dos principais problemas bucais enfrentados pelas gestantes são as doenças periodontais ${ }^{4-8}$, houve uma baixa proporção de raspagens gengivais relatadas. Como os dados avaliados por este estudo são oriundos dos relatos dos estudantes e sua percepção sobre o cuidado, não é possível determinar a causa para a baixa realização de determinados procedimentos.

Durante a gestação, o medo provocado pelo atendimento dentário aumenta pelo fato de se acreditar que hemorragias pós-extrações e uso de medicações seja prejudicial ao feto e à gravidez, levando a gestante a interromper o tratamento ou até mesmo não realizá- $10^{20}$. Nos dias atuais, a gestante não é mais considerada como um paciente de alto risco para o tratamento odontológico, com exceção daquelas em que se encontram em situação de gravidez de alto risco ${ }^{10-14,20-23}$. Todo tratamento odontológico pode ser realizado durante a gravidez, e apesar de não existirem meses proibitivos para esse atendimento, dá-se preferência para o segundo trimestre de gestação ${ }^{21}$. Por outro lado, reabilitações extensas e cirurgias eletivas, devem ser postergadas para depois do parto, evitando-se o desconforto de tempos prolongados na cadeira odontológica $^{14,22}$.

Enquanto $72 \%$ dos estudantes relataram não ter encontrado dificuldade durante o atendimento das gestantes, $43 \%$ afirmaram sentirem-se preparados para atender esse grupo, e apenas uma pequena parcela acertou quais os cuidados necessários a serem tomados no tratamento odontológico, como prescrição medicamentosa e uso de anestésicos. Somente $33 \%$ acertaram, por exemplo, o anestésico mais seguro para a gestante, enquanto $11 \%$ indicaram a prescrição de diclofenaco de sódio, sendo que anti-inflamatórios devem ser evitados durante toda a gestação. Essa análise permite descriminar quais alunos estão aptos para exercer o cuidado da gestante.

As evidências científicas demonstram que, na gestação, o anestésico mais indicado é a lidocaína a $2 \%$ com adrenalina 1.100 .000 , com o limite máximo de 2 tubetes $(3,6 \mathrm{ml})$ a serem utilizados por sessão $0^{21,22}$. Uma pequena parcela dos alunos respondeu que deveria ser utilizado qualquer anestésico sem vasoconstritor. Contudo, anestésicos sem vasoconstritor devem ser utilizados somente em procedimentos curtos, e pouco invasivos. Tais anestésicos podem não ser eficazes e seu efeito passará mais rapidamente $^{23}$. Outros alunos responderam que o anestésico indicado seria a Prilocaína, no entanto o uso deste sal próximo ao parto pode causar cianose por diminuição do oxigênio no sangue do recém-nascido ${ }^{24}$. Em relação à Mepivacaína, sugere-se que mais pesquisas sejam realizadas, já que seus riscos para o feto ainda não estão bem descritos na literatura e, portanto, seu uso é desaconselhado ${ }^{25}$.

Pouco mais da metade dos estudantes responderam que a gestante pode se submeter à exame radiográfico odontológico. Não é necessário evitar exame radiográfico no período gestacional, pois a radiação que a mãe é exposta para a tomada radiográfica periapical é muito menor que a dose necessária para causar malformações congênitas, pois o feto recebe $2 \%$ da radiação que é incidida diretamente na cabeça da mãe ${ }^{26}$.

Segundo o British Columbia Ministry and Nuclear Regulatory Commission, a quantidade de radiação absorvida pelo feto não deve exceder 
5 rads. Para obter essa quantidade de radiação, seriam necessárias 500 mil tomadas radiográficas odontológicas, correspondendo às radiografias periapicais ou interproximais ${ }^{27}$. Apenas radiações acima de 250 rads, antes de 16 semanas de gestação, oferecem risco para o desenvolvimento de malformações do feto, tais como microcefalia, microftalmia, retardo mental e catarata. O Colégio Americano de Obstetrícia e Ginecologia reconhece que a exposição limitada de raios $\mathrm{x}$ médicos para fins de diagnóstico não coloca o feto em risco ${ }^{21}$.

Algumas precauções na utilização de radiografias odontológicas garantem a segurança da mãe e do bebê, em qualquer estágio de gravidez $^{21}$ : direcionar o feixe diretamente para a boca, longe da barriga; orientar o uso de avental e protetor de tireoide para neutralizar os efeitos de radiação; escolher filmes de alta sensibilidade, permitindo um curto tempo de exposição $(0,2$ a $0,3 \mathrm{~s}$ ); e usar colimador do feixe de raios $\mathrm{X}$.

O padrão de prescrição de medicamentos para gestantes relatado pelos alunos aproximouse daquele observado entre médicos e cirurgiõesdentistas, coincidindo com os medicamentos mais usualmente prescritos na clínica odontológica. Em um inquérito realizado em hospitais do município do Rio Janeiro, foi encontrada a média de 2,3 medicamentos usados durante a gestação, sendo os mais prescritos anestésicos, antibióticos, ocitocina e analgésicos, com diferenças significativas entre estratos, e os mais referidos sulfato ferroso, vitaminas, escopolamina e paracetamol ${ }^{29}$. No presente estudo, grande parte dos alunos indicaram o paracetamol para controle da dor. Este medicamento é o mais prescrito por cirurgiões dentistas e é o analgésico de primeira escolha para gestantes ${ }^{28}$. Em relação à classe dos antibióticos, 119 (39\%) alunos recomendaram a amoxicilina. As penicilinas (classe a qual a amoxicilina pertence) são muito utilizadas em grávidas e apresentam ampla margem de segurança para a gestante e para o feto ${ }^{23}$. Vinte e oito alunos $(9 \%)$ marcaram o Metronidazol como sendo um medicamento possível de ser prescrito para as gestantes, porém o este antibiótico não deve ser utilizado durante a gestação e lactação, pois é potencialmente teratogênico para seres humanos ${ }^{12,14}$. Apenas dois alunos citaram a Tetraciclina como sendo indicada para gestantes, no entanto as Tetraciclinas quando utilizadas até a segunda metade da gestação causam hipoplasia dos dentes o dos ossos do feto e foram associadas à ocorrência de catarata congênita. As Tetraciclinas atravessam facilmente a placenta e pertencem ao grupo D, na classificação da Food and Drug Administration (FDA) ${ }^{23}$.

Moimaz e colaboradores avaliaram a percepção de estudantes da Faculdade de Odontologia de Araçatuba (UNESP) sobre a participação em um projeto extensionista de cuidado odontológico na gravidez, a eficácia das ações realizadas e o alcance das metas propostas $^{30}$. Os autores relataram que metade dos alunos considerou a prescrição medicamentosa como o tema de maior dificuldade durante o atendimento odontológico à gestante. Este resultado está em consonância com os achados deste estudo, já que $46 \%$ dos alunos não souberam responder qual medicamento poderia ser prescrito para a gestante. Em outro trabalho do mesmo grupo de pesquisadores, foi observado que $70,5 \%$ dos estudantes foram capazes de indicar corretamente o anestésico local para uso odontológico em gestante, enquanto no presente estudo a taxa de acerto foi de $33 \%{ }^{31}$. Há uma discrepância também quanto ao conhecimento referente ao período ideal para realização do tratamento odontológico em gestantes, sendo que $84,8 \%$ dos alunos daquele grupo responderam corretamente ser no segundo trimestre, enquanto neste apenas $56,5 \%$ acertaram a mesma questão. O Programa de Atenção Odontológica à Gestante 
da Faculdade de Odontologia de Araçatuba está inserido no curso de graduação como projeto de extensão e disciplina extramural, e proporciona aos graduandos a oportunidade de atender gestantes em uma clínica criada especificamente para esse fim, além de participarem de atividades de educação em saúde voltadas para esse público $^{30,31}$. Percebe-se o impacto dessa formação ao se comparar as taxas de acerto das respostas dos alunos de Araçatuba com os participantes desta pesquisa.

Ainda hoje é comum a frequência de crenças populares que proferem que gestantes não devem receber assistência odontológica, sob o risco de prejudicar a mãe ou o bebê. É dever do cirurgião-dentista ou qualquer profissional da saúde desmistificar tais crenças e adágios, atendendo a paciente gestante ou fazendo o encaminhamento para os serviços de saúde bucal ${ }^{10,24}$. Para isso, é necessária a formação adequada durante o curso de graduação. O preparo apropriado do profissional em relação às alterações sistêmicas da gravidez, saúde e desenvolvimento do feto, além de informações sobre os fármacos e anestésicos indicados para esse período, podem colaborar na realização de um atendimento tranquilo, eficaz e seguro, no fortalecimento do vínculo profissional-paciente, com consequências positivas sobre as ações de educação e prevenção em saúde, e no empoderamento da gestante no cuidado de sua própria saúde e de seu bebê $\hat{e}^{23}$.

Este trabalho tem como ponto forte o ineditismo de seus resultados, visto a carência de estudos sobre essa temática. Além disso, apresenta impacto relevante na formulação de políticas de ensino com o objetivo de melhorar a formação do cirurgião-dentista para a assistência no pré-natal.

Dentre suas fragilidades está o viés de resposta, sob duas perspectivas. Primeira, a perda de respondentes pode levar a um resultado espúrio, já que não se sabe se aqueles que não participaram poderiam ser estudantes mais conscientes da temática estudada. Em segundo plano, por tratar-se de uma abordagem retrospectiva, têm-se que os participantes estavam mais sujeitos ao viés de memória, que pode ter induzido maiores erros nas respostas, e levando à constatação de que o assunto não é tratado durante o curso de graduação. É desafiador trabalhar com amostras grandes em ambientes universitários, por causa da mobilidade acadêmica dos estudantes. Além disso, é difícil estipular em qual período exato o aluno se encontra, já que muitos estão matriculados em disciplinas de mais de um período específico.

\section{CONCLUSÕES}

Os estudantes de odontologia da instituição de ensino avaliada apresentaram deficiências no conhecimento sobre o atendimento odontológico à gestante, e demonstraram interesse em melhorar seu aprendizado nessa área. As oportunidades de atendimento odontológico de uma paciente gestante foram pequenas neste curso de graduação em Odontologia, e muitos estudantes não se sentiam preparados para exercer o cuidado na gravidez. Assim, os resultados deste estudo sugerem que o ensino da graduação nessa instituição não está formando adequadamente os alunos para o atendimento odontológico da gestante, reforçando o ciclo de medos e adágios que envolvem o pré-natal odontológico, havendo necessidade de reformulações curriculares ou ações educacionais com o intuito de capacitar o graduando para atuar em equipes de saúde nos serviços de pré-natal.

\section{AGRADECIMENTOS}

Agradecemos o apoio financeiro da Pró-Reitoria de Pesquisa da Universidade Federal de Minas 
Gerais (PRPq) através do edital Programa institucional de auxílio à pesquisa de docentes recém-contratados ou recém-doutorados EDITAL PRPq - 05/2016.

\section{ABSTRACT \\ Dental care during pregnancy: knowledge and perceptions among Dentistry students}

Dental treatment during pregnancy is surrounded by myths and uncertainties, perpetuated by the fear of treating pregnant women. Few dental schools in Brazil include some disciplines, projects, programs, or actions that prepare undergraduate students for providing dental care to pregnant women. The aim of this study was to assess the knowledge of undergraduate dental students about the dental care of pregnant women and the needs of these students to learn about this topic. A semistructured questionnaire was applied to all students enrolled for the second semester of 2016 at the Dental School of Universidade Federal de Minas Gerais. The questionnaire contained questions about knowledge and expectations of dental treatment of pregnant women. The results were evaluated by content analysis and prevalence estimates were calculated. Among the 303 students who answered the questionnaire, only $14 \%$ attended to pregnant women during their undergraduate course. Only $43 \%$ of the students thought they were prepared to treat pregnant women and 62\% reported having been given some guidelines on prenatal dental care during their undergraduate studies, with no formal syllabus dealt with in any discipline. Regarding technical qualification, the major problems were lack of knowledge about the use of medications, anesthetics, and procedures. In conclusion, students lacked knowledge about dental treatment of pregnant women, but they showed interest in improving their learning in this field. Undergraduate courses do not properly qualify students for prenatal dental care, strengthening the fears and aphorisms surrounding this healthcare service.

Descriptors: Pregnant Women. Dental Care. Knowledge. Students. Dental Education.

\section{REFERÊNCIAS}

1. Figueiredo CSA, Rosalem CGC, Catanhede ALC, Thomaz EBAF, Cruz MCFN. Systemic alterations and their oral manifestations in pregnant women. J Obstet Gynaecol Res. 2017; 43(1):16-22.

2. Jessani A, Laronde D, Mathu-Muju K, Brondani MA. Self-Perceived Oral Health and Use of Dental Services by Pregnant Women in Surrey, British Columbia. J Can Dent Assoc. 2016; 82:g28.

3. Amar S, Chung KM. Influence of hormonal variation on the periodontium in women. Periodontol 2000. 1994; 6:79-87.

4. Fujiwara N, Tsuruda K, Iwamoto Y, Kato F, Odaki T, Yamane $\mathrm{N}$ et al. Significant increase of oral bacteria in the early pregnancy period in Japanese women. $\mathrm{J}$ Investig Clin Dent. 2017; 8(1):1-8.

5. Onigbinde O, Sorunke $\mathrm{M}$, Braimoh $\mathrm{M}$, Adeniyi A. Periodontal status and some variables among pregnant women in a Nigeria Tertiary Institution. Ann Med Health Sci Res. 2014; 4(6):852-7.

6. González-Jaranay M, Téllez L, Roa-López A, Gómez-Moreno G, Moreu G. Periodontal status during pregnancy and postpartum. PLoS One. 201719; 12(5): e0178234.

7. Moimaz SAS, Garbin CAS, Saliba NA, Zina LG. Condição periodontal durante a gestação em um grupo de mulheres brasileiras. Ciênc Odontol Bras. 2006; 9(4):59-66.

8. Schwendicke F, Karimbux N, Allareddy V, Gluud C. Periodontal treatment for preventing adverse pregnancy outcomes: a meta- and trial sequential analysis. PLoS One. 2015; 10(6):1-12. 
9. Krüger MSM, Lang CA, Almeida LHS, Bello-Corrêa FO, Romano AR, Pappen FG. Dental pain and associated factors among pregnant women: an observational study. Matern Child Health J. 2015; 19(3): 504-10.

10. Rocha NB, Rovida TAS, Moimaz SAS. Crendices e adágios populares em relação ao atendimento odontológico às pacientes gestantes. In: Moimaz SAS, Saliba N, Garbin CAS. Odontologia para gestante: guia para o profissional de saúde. Araçatuba: Programa de pós-graduação em Odontologia Preventiva e Social - FOA/UNESP; 2009. p. 35-40.

11. Codato LAB, Nakama L, Júnior L C, Higasi M S. Atenção Odontológica à gestante: papel dos profissionais de saúde. Ciênc Saúde Colet. 2011; 16(4): 2297-301.

12. Echeverria S, Politano GT. Tratamento odontológico para gestantes. $2^{\mathrm{a}}$.ed. São Paulo: Santos; 2014.

13. Brasil. Diretrizes da Política Nacional de Saúde Bucal. Brasília: Ministério da Saúde; 2004 [Acesso em 02 ago. 2017]. Disponível em: http://bvsms.saude.gov.br/bvs/publica coes/politica_nacional_brasil_sorridente.pdf.

14. Bertness J, Holt K. Oral health care during pregnancy: a resource guide. Washington (D.C.): National Maternal and Child Oral Health Resource Center; 2012.

15. Moimaz SAS, Saliba NA, Garbin, CAS, Zina LG, Moscardini TM. Avaliação da percepção de acadêmicos de odontologia sobre a participação no Programa de Atenção Odontológica à Gestante da FOAUNESP. Rev Ciênc Ext. 2006; 2(2):115.

16. Kloetzel MK, Huebner CE, Milgrom $\mathrm{P}$, Littell CT, Eggertsson H. Oral health in pregnancy: educational needs of dental professionals and office staff. J Public Health Dent. 2012; 72(4):279-86.
17. Bradburn N, Sudman S, Wansink B. Asking Questions: The definitive guide to questionnaire design for market research, political polls, and health questionnaires (Revised Edition). San Francisco: Josse Bass; 2004.

18. Bardin L. Análise de Conteúdo. $3^{\mathrm{a}}$ ed. São Paulo: Ed. 70; 2004.

19. Gokmen Karasu AF, Kutuk N, Aydin S, Adanir I, Ates S, Bademler N. Dental health dispositions of pregnant women: A survey from a hospital clinic in Istanbul. J Obstet Gynaecol. 2017; 37(6):752-6.

20. Figueira TR, Ferreira E, Schall V, Moderna C. O modelo de crenças e saúde e o processo saúde-doença-cuidado bucal por gestantes. ROBRAC. 2013; 22(63):169-73.

21. Fagoni TG, Vasconcelos RA, Cardoso PE, Gomes APM, Camargo HR, Gonçalves SHF. Dental treatment for the pregnant patient. Braz Dent Sci. 2014; 17(3):3-10.

22. Hemalatha VT, Manigandan T, Sarumathi T, Aarthi Nish V, Amudhan A. Dental considerations in pregnancy-a critical review on the oral care. J Clin Diagn Res. 2013; 7(5):948-953.

23. Bastos RDS, Silva BS, Cardoso JA, Farias JG, Falcão GGVCS. Desmistificando o atendimento odontológico à gestante. Rev Bahiana Odontol. 2014; 5(2):104-16.

24. Caneppele TMF, Yamamoto EC, Souza AC, Valeria MC, Araújo MAM. Conhecimento dos cirurgiões-dentistas sobre o atendimento de pacientes especiais: hipertensos, diabéticos e gestantes. J Biodent Biomaterials. 2011; (1):31-41.

25. Fayans EP, Stuart HR, Carsten D, Ly Q, Kim H. Local anesthetic use in the pregnant and postpartum patient. Dent Clin North Am. 2010; 54(4):697-713.

26. Silva SZO. Pré natal odontológico: A importância da educação em saúde para a 
promoção da saúde bucal no período gestacional [especialização]. Belo Horizonte (MG): Universidade Federal de Minas Gerais; 2013.

27. Varellis MLZ. O paciente com necessidades especiais na odontologia. $3^{\text {a }}$. ed. São Paulo: Santos; 2017.

28. Amadei SU, Carmo ED, Pereira AS, Silveira VAS, Rocha RF. Prescrição medicamentosa no tratamento odontológico de grávidas e lactantes. RGO. 2011; 59(Supl 1):31-7.

29. Osorio-de-Castro CGS, Pepe VLE, Luiza VL, Cosendey MAE, Freitas AM Miranda FF et al. Uso indicado e uso referido de medicamentos durante a gravidez. Cad Saúde Pública 2004; 20(Suppl 1):S73-S82.
30. Moimaz SAS, Saliba NA, Garbin, CAS, Zina LG, Moscardini TM. Avaliação da percepção de acadêmicos de odontologia sobre a participação no Programa de Atenção Odontológica à Gestante da FOAUNESP. Rev Ciênc Ext. 2006; 2(2):7891.

31. Garbin CAS, Garbin AJI, Moimaz SAS, Lelis RT. Odontologia para gestante: Avaliação do conhecimento de acadêmicos da Faculdade de Odontologia de AraçatubaUNESP. J Bras Clin Odontol Integr. 2006; 10(53):155-61.

Correspondência para:

Lívia Guimarães Zina

e-mail: liviazina@ufmg.br

Departamento de Odontologia Social e Preventiva, Faculdade de Odontologia/UFMG. Av. Antônio Carlos, 6627 - Pampulha 31270-901 Belo Horizonte/MG 